\title{
Associative Space-Time Sedenions and Their Application in Relativistic Quantum Mechanics and Field Theory
}

\author{
Victor L. Mironov ${ }^{1,2}$, Sergey V. Mironov ${ }^{1}$ \\ ${ }^{1}$ Institute for Physics of Microstructures RAS, Nizhny Novgorod, Russia \\ ${ }^{2}$ Lobachevsky State University of Nizhny Novgorod, Nizhny Novgorod, Russia \\ Email: mironov@ipmras.ru
}

Received 24 October 2014; revised 20 November 2014; accepted 15 December 2014

Copyright (C) 2015 by authors and Scientific Research Publishing Inc.

This work is licensed under the Creative Commons Attribution International License (CC BY).

http://creativecommons.org/licenses/by/4.0/

(c) (i) Open Access

\begin{abstract}
We present an alternative sixteen-component hypercomplex scalar-vector values named "spacetime sedenions", generating associative noncommutative space-time Clifford algebra. The generalization of relativistic quantum mechanics and field theory equations based on sedenionic wave function and space-time operators is discussed.
\end{abstract}

\section{Keywords}

Clifford Algebra, Space-Time Sedenions, Relativistic Quantum Mechanics, Sedenionic Klein-Gordon Equation, Sedenionic Dirac Equation, Sedenionic Maxwell Equiations

\section{Introduction}

The multicomponent hypercomplex numbers such as quaternions and octonions are widely used for the reformulation of quantum mechanics and field theory equations. The first generalization of quantum mechanics and electrodynamics was made on the basis of four-component quaternions, which were interpreted as scalar-vector structures [1]-[5]. The next step was taken on the basis of eight-component octonions, which were interpreted as the sum of scalar, pseudoscalar, polar vector and axial vector [6]-[11]. Scalars and axial vectors are not transformed under spatial inversion, while pseudoscalars and polar vectors change their sign under spatial inversion. Therefore, this interpretation takes only the symmetry with respect to the spatial inversion into account. However, a consistent relativistic approach requires taking full time and space symmetries into consideration that leads to the sixteen-component space-time algebras.

The well-known sixteen-component hypercomplex numbers, sedenions, are obtained from octonions by the 
Cayley-Dickson extension procedure [12] [13]. In this case the sedenion is defined as

$$
S=O_{1}+O_{2} \boldsymbol{e}
$$

where $O_{i}$ is an octonion and the parameter of duplication $\boldsymbol{e}$ is similar to imaginary unit $\boldsymbol{e}^{2}=-1$. The algebra of sedenions has the specific rules of multiplication. The product of two sedenions

$$
\begin{aligned}
& S_{1}=O_{11}+O_{12} \boldsymbol{e}, \\
& S_{2}=O_{21}+O_{22} \boldsymbol{e},
\end{aligned}
$$

is defined as

$$
S_{1} S_{2}=\left(O_{11}+O_{12} \boldsymbol{e}\right)\left(O_{21}+O_{22} \boldsymbol{e}\right)=\left(O_{11} O_{21}-\bar{O}_{22} O_{12}\right)+\left(O_{22} O_{11}+O_{12} \bar{O}_{21}\right) \boldsymbol{e},
$$

where $\bar{O}_{i j}$ is conjugated octonion. The sedenionic multiplication (2) allows one to introduce a well-defined norm of sedenion. However, such procedure of constructing the higher hypercomplex numbers leads to the fact that the sedenions as well as octonions generate normed but nonassociative algebra [14]-[16]. It complicates the use of the Cayley-Dickson sedenions in the physical applications.

Recently we have developed an alternative approach to constructing the multicomponent values based on our scalar-vector conception realized in associative eight-component octons [17]-[19] and sixteen-component sedeons [20]-[24]. In particular, we have demonstrated the method, which allows one to reformulate the equations of relativistic quantum mechanics and field theory on the basis of sedeonic space-time operators and scalar-vector wave functions. In this paper we present an alternative version of the sixteen-component associative space-time hypercomplex algebra and demonstrate some of its application to the generalization of relativistic quantum mechanics and field theory equations.

\section{Sedenionic Space-Time Algebra}

It is known, the quaternion is a four-component object

$$
\widehat{q}=q_{0} \mathbf{a}_{0}+q_{1} \mathbf{a}_{1}+q_{2} \mathbf{a}_{2}+q_{3} \mathbf{a}_{3},
$$

where components $q_{v}$ (Greek indexes $v=0,1,2,3$ ) are numbers (complex in general), $\mathbf{a}_{0} \equiv 1$ is scalar units and values $\mathbf{a}_{\mathbf{m}}$ (Latin indexes $m=1,2,3$ ) are quaternionic units, which are interpreted as unit vectors. The rules of multiplication and commutation for $\mathbf{a}_{\mathbf{m}}$ are presented in Table 1. We introduce also the space-time basis $\mathbf{e}_{\mathbf{t}}, \mathbf{e}_{\mathbf{r}}$, $\mathbf{e}_{\mathbf{t r}}$, which is responsible for the space-time inversions. The indexes $\mathbf{t}$ and $\mathbf{r}$ indicate the transformations (t for time inversion and $\mathbf{r}$ for spatial inversion), which change the corresponding values. The value $\mathbf{e}_{\mathbf{0}} \equiv 1$ is a scalar unit. For convenience we introduce numerical designations $\mathbf{e}_{1} \equiv \mathbf{e}_{\mathbf{t}}$ (time scalar unit); $\mathbf{e}_{2} \equiv \mathbf{e}_{\mathbf{r}}$ (space scalar unit) and $\mathbf{e}_{3} \equiv \mathbf{e}_{\text {tr }}$ (space-time scalar unit). The rules of multiplication and commutation for this basis we choose similar to the rules for quaternionic units (see Table 2).

\begin{tabular}{cccc}
\multicolumn{4}{l}{ Table 1. Multiplication rules for unit vectors $\mathbf{a}_{\mathbf{m}}$. } \\
\hline & $\mathbf{a}_{1}$ & $\mathbf{a}_{2}$ & $\mathbf{a}_{3}$ \\
\hline $\mathbf{a}_{1}$ & -1 & $\mathbf{a}_{3}$ & $-\mathbf{a}_{2}$ \\
$\mathbf{a}_{2}$ & $-\mathbf{a}_{2}$ & -1 & $\mathbf{a}_{1}$ \\
$\mathbf{a}_{3}$ & $\mathbf{a}_{2}$ & $-\mathbf{a}_{1}$ & -1 \\
\hline & & & \\
\hline Table 2. Multiplication rules for space-time units. & \\
\hline & $\mathbf{e}_{1}$ & $\mathbf{e}_{2}$ & $\mathbf{e}_{3}$ \\
\hline $\mathbf{e}_{1}$ & -1 & $\mathbf{e}_{3}$ & $-\mathbf{e}_{2}$ \\
$\mathbf{e}_{2}$ & $-\mathbf{e}_{2}$ & -1 & $\mathbf{e}_{1}$ \\
$\mathbf{e}_{3}$ & $\mathbf{e}_{2}$ & $-\mathbf{e}_{1}$ & -1 \\
\hline
\end{tabular}


Note that the unit vectors $\mathbf{a}_{1}, \mathbf{a}_{2}, \mathbf{a}_{3}$ and the space-time units $\mathbf{e}_{\mathbf{1}}, \mathbf{e}_{2}, \mathbf{e}_{3}$ generate the anticommutative algebras:

$$
\begin{aligned}
& \mathbf{a}_{n} \mathbf{a}_{m}=-\mathbf{a}_{m} \mathbf{a}_{n}, \\
& \mathbf{e}_{n} \mathbf{e}_{m}=-\mathbf{e}_{m} \mathbf{e}_{n},
\end{aligned}
$$

for $\mathbf{n} \neq \mathbf{m}$, but $\mathbf{e}_{1}, \mathbf{e}_{2}, \mathbf{e}_{3}$ commute with $\mathbf{a}_{1}, \mathbf{a}_{2}, \mathbf{a}_{3}$ :

$$
\mathbf{e}_{\mathrm{n}} \mathbf{a}_{\mathrm{m}}=\mathbf{a}_{\mathrm{m}} \mathbf{e}_{\mathrm{n}}
$$

for any $\mathbf{n}$ and $\mathbf{m}$. Besides, we assume the associativity of $\mathbf{e}_{1}, \mathbf{e}_{2}, \mathbf{e}_{3}, \mathbf{a}_{1}, \mathbf{a}_{2}, \mathbf{a}_{3}$ multiplication.

Then we can introduce the sixteen-component space-time sedenion $\tilde{\boldsymbol{V}}$ in the following form:

$$
\begin{aligned}
\tilde{V}= & \mathbf{e}_{\mathbf{0}}\left(V_{00} \mathbf{a}_{\mathbf{0}}+V_{01} \mathbf{a}_{\mathbf{1}}+V_{02} \mathbf{a}_{\mathbf{2}}+V_{03} \mathbf{a}_{\mathbf{3}}\right)+\mathbf{e}_{\mathbf{1}}\left(V_{10} \mathbf{a}_{\mathbf{0}}+V_{11} \mathbf{a}_{\mathbf{1}}+V_{12} \mathbf{a}_{\mathbf{2}}+V_{13} \mathbf{a}_{\mathbf{3}}\right) \\
& +\mathbf{e}_{\mathbf{2}}\left(V_{20} \mathbf{a}_{\mathbf{0}}+V_{21} \mathbf{a}_{\mathbf{1}}+V_{22} \mathbf{a}_{\mathbf{2}}+V_{23} \mathbf{a}_{\mathbf{3}}\right)+\mathbf{e}_{\mathbf{3}}\left(V_{30} \mathbf{a}_{\mathbf{0}}+V_{31} \mathbf{a}_{\mathbf{1}}+V_{32} \mathbf{a}_{\mathbf{2}}+V_{33} \mathbf{a}_{\mathbf{3}}\right) .
\end{aligned}
$$

The sedenionic components $V_{v \mu}$ are numbers (complex in general). Introducing designation of scalar and vector values in accordance with the following relations

$$
\begin{aligned}
& V=\mathbf{e}_{\mathbf{0}} V_{00} \mathbf{a}_{\mathbf{0}}, \\
& \vec{V}=\mathbf{e}_{\mathbf{0}}\left(V_{01} \mathbf{a}_{\mathbf{1}}+V_{02} \mathbf{a}_{\mathbf{2}}+V_{03} \mathbf{a}_{\mathbf{3}}\right), \\
& V_{\mathbf{t}} \equiv V_{\mathbf{1}}=\mathbf{e}_{\mathbf{1}} V_{10} \mathbf{a}_{\mathbf{0}}, \\
& \vec{V}_{\mathbf{t}} \equiv \vec{V}_{\mathbf{1}}=\mathbf{e}_{\mathbf{1}}\left(V_{11} \mathbf{a}_{\mathbf{1}}+V_{12} \mathbf{a}_{\mathbf{2}}+V_{13} \mathbf{a}_{\mathbf{3}}\right), \\
& V_{\mathbf{r}} \equiv V_{\mathbf{2}}=\mathbf{e}_{\mathbf{2}} V_{20} \mathbf{a}_{\mathbf{0}}, \\
& \vec{V}_{\mathbf{r}} \equiv \vec{V}_{\mathbf{2}}=\mathbf{e}_{\mathbf{2}}\left(V_{21} \mathbf{a}_{\mathbf{1}}+V_{22} \mathbf{a}_{\mathbf{2}}+V_{23} \mathbf{a}_{\mathbf{3}}\right), \\
& V_{\mathbf{t r}} \equiv V_{\mathbf{3}}=\mathbf{e}_{3} V_{30} \mathbf{a}_{\mathbf{0}}, \\
& \vec{V}_{\mathbf{t r}} \equiv \vec{V}_{\mathbf{3}}=\mathbf{e}_{\mathbf{3}}\left(V_{31} \mathbf{a}_{\mathbf{1}}+V_{32} \mathbf{a}_{\mathbf{2}}+V_{33} \mathbf{a}_{\mathbf{3}}\right) .
\end{aligned}
$$

we can represent the sedenion in the following scalar-vector form:

$$
\tilde{\boldsymbol{V}}=V+\vec{V}+V_{\mathbf{t}}+\vec{V}_{\mathbf{t}}+V_{\mathbf{r}}+\vec{V}_{\mathbf{r}}+V_{\mathbf{t r}}+\vec{V}_{\mathbf{t r}} .
$$

Thus, the sedenionic algebra encloses four groups of values, which are differed with respect to spatial and time inversion.

1) Absolute scalars $(V)$ and absolute vectors $(\vec{V})$ are not transformed under spatial and time inversion.

2) Time scalars $\left(V_{t}\right)$ and time vectors $\left(\vec{V}_{\mathbf{t}}\right)$ are changed (in sign) under time inversion and are not transformed under spatial inversion.

3) Space scalars $\left(V_{\mathbf{r}}\right)$ and space vectors $\left(\vec{V}_{\mathbf{r}}\right)$ are changed under spatial inversion and are not transformed under time inversion.

4) Space-time scalars $\left(V_{\mathrm{tr}}\right)$ and space-time vectors $\left(\vec{V}_{\mathrm{tr}}\right)$ are changed under spatial and time inversion.

Further we will use the symbol 1 instead units $\mathbf{a}_{\mathbf{0}}$ and $\mathbf{e}_{\mathbf{0}}$ for simplicity. Introducing the designations of scalarvector values

$$
\begin{aligned}
& \overline{\boldsymbol{V}}_{0}=V_{00}+V_{01} \mathbf{a}_{1}+V_{\odot 2} \mathbf{a}_{2}+V_{03} \mathbf{a}_{3}, \\
& \overline{\boldsymbol{V}}_{1}=V_{10}+V_{11} \mathbf{a}_{1}+V_{12} \mathbf{a}_{2}+V_{13} \mathbf{a}_{3}, \\
& \overline{\boldsymbol{V}}_{2}=V_{20}+V_{21} \mathbf{a}_{1}+V_{22} \mathbf{a}_{2}+V_{23} \mathbf{a}_{3}, \\
& \overline{\boldsymbol{V}}_{3}=V_{30}+V_{31} \mathbf{a}_{1}+V_{32} \mathbf{a}_{2}+V_{33} \mathbf{a}_{3} .
\end{aligned}
$$

we can write the sedenion (6) in the following compact form:

$$
\tilde{\boldsymbol{V}}=\overline{\boldsymbol{V}}_{0}+\mathbf{e}_{\mathbf{1}} \overline{\boldsymbol{V}}_{1}+\mathbf{e}_{2} \overline{\boldsymbol{V}}_{2}+\mathbf{e}_{\mathbf{3}} \overline{\boldsymbol{V}}_{3} .
$$

On the other hand, introducing designations of space-time sedenion-scalars

we can write the sedenion (6) as

$$
\begin{aligned}
& \boldsymbol{V}_{0}=\left(V_{00}+\mathbf{e}_{\mathbf{1}} V_{10}+\mathbf{e}_{2} V_{20}+\mathbf{e}_{\mathbf{3}} V_{30}\right), \\
& \boldsymbol{V}_{1}=\left(V_{01}+\mathbf{e}_{\mathbf{1}} V_{11}+\mathbf{e}_{2} V_{21}+\mathbf{e}_{3} V_{31}\right), \\
& \boldsymbol{V}_{2}=\left(V_{02}+\mathbf{e}_{\mathbf{1}} V_{12}+\mathbf{e}_{\mathbf{2}} V_{22}+\mathbf{e}_{\mathbf{3}} V_{32}\right), \\
& \boldsymbol{V}_{3}=\left(V_{03}+\mathbf{e}_{\mathbf{1}} V_{13}+\mathbf{e}_{\mathbf{2}} V_{23}+\mathbf{e}_{\mathbf{3}} V_{33}\right) .
\end{aligned}
$$




$$
\tilde{\boldsymbol{V}}=\boldsymbol{V}_{0}+\boldsymbol{V}_{1} \mathbf{a}_{1}+V_{2} \mathbf{a}_{2}+V_{3} \mathbf{a}_{3}
$$

or introducing the sedenion-vector

$$
\vec{V}=\vec{V}+\vec{V}_{\mathbf{t}}+\vec{V}_{\mathbf{r}}+\vec{V}_{\mathbf{t r}}=\boldsymbol{V}_{1} \mathbf{a}_{1}+\boldsymbol{V}_{2} \mathbf{a}_{2}+\boldsymbol{V}_{3} \mathbf{a}_{3},
$$

we can rewrite the sedenion in following compact form:

$$
\tilde{\boldsymbol{V}}=\boldsymbol{V}_{0}+\overrightarrow{\boldsymbol{V}}
$$

Further we will indicate sedenion-scalars and sedenion-vectors with the bold capital letters.

Let us consider the sedenionic multiplication in detail. The sedenionic product of two sedenions $\tilde{\boldsymbol{A}}$ and $\tilde{\boldsymbol{B}}$ can be represented in the following form

$$
\tilde{A} \tilde{B}=\left(A_{0}+\vec{A}\right)\left(B_{0}+\vec{B}\right)=A_{0} B_{0}+A_{0} \vec{B}+\vec{A} B_{0}+(\vec{A} \cdot \vec{B})+[\vec{A} \times \vec{B}] .
$$

Here we denoted the sedenionic scalar multiplication of two sedenion-vectors (internal product) by symbol “.” and round brackets

$$
(\vec{A} \cdot \vec{B})=-A_{1} B_{1}-A_{2} B_{2}-A_{3} B_{3},
$$

and sedenionic vector multiplication (external product) by symbol " $\times$ " and square brackets,

$$
[\vec{A} \times \vec{B}]=\left(A_{2} B_{3}-A_{3} B_{2}\right) \mathbf{a}_{1}+\left(A_{3} B_{1}-A_{1} B_{3}\right) \mathbf{a}_{2}+\left(A_{1} B_{2}-A_{2} B_{1}\right) \mathbf{a}_{3} .
$$

In (16) and (17) the multiplication of sedenionic components is performed in accordance with (11) and Table 2. Thus the sedenionic product

$$
\tilde{\boldsymbol{F}}=\tilde{\boldsymbol{A}} \tilde{\boldsymbol{B}}=\boldsymbol{F}_{0}+\overrightarrow{\boldsymbol{F}},
$$

has the following components:

$$
\begin{aligned}
& F_{0}=A_{0} B_{0}-A_{1} B_{1}-A_{2} B_{2}-A_{3} B_{3}, \\
& F_{1}=A_{1} B_{0}+A_{0} B_{1}+\left(A_{2} B_{3}-A_{3} B_{2}\right), \\
& F_{2}=A_{2} B_{0}+A_{0} B_{2}+\left(A_{3} B_{1}-A_{1} B_{3}\right), \\
& F_{3}=A_{3} B_{0}+A_{0} B_{3}+\left(A_{1} B_{2}-A_{2} B_{1}\right) .
\end{aligned}
$$

Note that in the sedenionic algebra the square of vector is defined as

$$
\vec{A}^{2}=(\vec{A} \cdot \vec{A})=-A_{1}^{2}-A_{2}^{2}-A_{3}^{2},
$$

and the square of modulus of vector is

$$
|\vec{A}|^{2}=-(\vec{A} \cdot \vec{A})=A_{1}^{2}+A_{2}^{2}+A_{3}^{2} .
$$

\section{Spatial Rotation and Space-Time Inversion}

The rotation of sedenion $\tilde{V}$ on the angle $\theta$ around the absolute unit vector $\vec{n}$ is realized by sedenion

$$
\tilde{U}=\cos (\theta / 2)+\vec{n} \sin (\theta / 2),
$$

and by conjugated sedenion $\tilde{\boldsymbol{U}}^{*}$ :

$$
\tilde{\boldsymbol{U}}^{*}=\cos (\theta / 2)-\vec{n} \sin (\theta / 2),
$$

with

$$
\tilde{\boldsymbol{U}} \tilde{\boldsymbol{U}}^{*}=\tilde{\boldsymbol{U}}^{*} \tilde{\boldsymbol{U}}=1 .
$$

The transformed sedenion $\tilde{\boldsymbol{V}}^{\prime}$ is defined as sedenionic product

$$
\tilde{\boldsymbol{V}}^{\prime}=\tilde{\boldsymbol{U}}^{*} \tilde{\boldsymbol{V}} \tilde{\boldsymbol{U}},
$$

Thus, the transformed sedenion $\tilde{\boldsymbol{V}}^{\prime}$ can be written as 


$$
\begin{aligned}
\tilde{\boldsymbol{V}}^{\prime} & =[\cos (\theta / 2)-\vec{n} \sin (\theta / 2)]\left(\boldsymbol{V}_{0}+\overrightarrow{\boldsymbol{V}}\right)[\cos (\theta / 2)+\vec{n} \sin (\theta / 2)] \\
& =\boldsymbol{V}_{0}+\overrightarrow{\boldsymbol{V}} \cos \theta-\vec{n}(\vec{n} \cdot \overrightarrow{\boldsymbol{V}})(1-\cos \theta)-[\vec{n} \times \overrightarrow{\boldsymbol{V}}] \sin \theta .
\end{aligned}
$$

It is clearly seen that rotation does not transform the sedenion-scalar part, but the sedenionic vector $\vec{V}$ is rotated on the angle $\theta$ around $\vec{n}$.

The operations of time inversion $\left(\hat{R}_{\mathrm{t}}\right)$, space inversion $\left(\hat{R}_{\mathrm{r}}\right)$ and space-time inversion $\left(\hat{R}_{\mathrm{tr}}\right)$ are connected with transformations in $\mathbf{e}_{1}, \mathbf{e}_{2}, \mathbf{e}_{3}$ basis and can be presented as

$$
\begin{aligned}
& \hat{R_{\mathbf{t}}} \tilde{\boldsymbol{V}}=-\mathbf{e}_{2} \tilde{\boldsymbol{V}} \mathbf{e}_{2}=\bar{V}_{0}-\mathbf{e}_{1} \bar{V}_{1}+\mathbf{e}_{2} \bar{V}_{2}-\mathbf{e}_{3} \bar{V}_{3}, \\
& \hat{R}_{\mathbf{r}} \tilde{V}=-\mathbf{e}_{1} \tilde{V} \mathbf{e}_{1}=\bar{V}_{0}+\mathbf{e}_{1} \bar{V}_{1}-\mathbf{e}_{2} \bar{V}_{2}-\mathbf{e}_{3} \bar{V}_{3}, \\
& \hat{R}_{\mathbf{t r}} \tilde{V}=-\mathbf{e}_{3} \tilde{\mathbf{V}_{3}}=\bar{V}_{0}-\mathbf{e}_{1} \bar{V}_{1}-\mathbf{e}_{2} \bar{V}_{2}+\mathbf{e}_{3} \bar{V}_{3} .
\end{aligned}
$$

\section{Sedenionic Lorentz Transformations}

The relativistic event four-vector can be represented in the follow sedenionic form:

$$
\tilde{\boldsymbol{S}}=\mathbf{e}_{1} c t+\mathbf{e}_{2} \vec{r} .
$$

The square of this valueis the Lorentzinvariant

$$
\tilde{\boldsymbol{S}} \tilde{\boldsymbol{S}}=-c^{2} t^{2}+x^{2}+y^{2}+z^{2} .
$$

The Lorentz transformation of event four-vector is realized by sedenions

$$
\begin{aligned}
& \tilde{\boldsymbol{L}}=\operatorname{ch} \vartheta+\mathbf{e}_{3} \vec{m} \operatorname{sh} \vartheta, \\
& \tilde{\boldsymbol{L}}^{*}=\operatorname{ch} \vartheta-\mathbf{e}_{3} \vec{m} \operatorname{sh} \vartheta,
\end{aligned}
$$

where th $2 \vartheta=v / c, \quad v$ is velocity of motion along the absolute unit vector $\vec{m}$. Note that

$$
\tilde{\boldsymbol{L}}^{*} \tilde{\boldsymbol{L}}=\tilde{\boldsymbol{L}} \tilde{\boldsymbol{L}}^{*}=1 .
$$

The transformed event four-vector $\tilde{\boldsymbol{S}}^{\prime}$ is written as

$$
\begin{aligned}
\tilde{\boldsymbol{S}}^{\prime} & =\tilde{\boldsymbol{L}}^{*} \tilde{\boldsymbol{S}} \tilde{\boldsymbol{L}}=\left(\operatorname{ch} \vartheta-\mathbf{e}_{3} \operatorname{sh} \vartheta \vec{m}\right)\left(\mathbf{e}_{1} c t+\mathbf{e}_{2} \vec{r}\right)\left(\operatorname{ch} \vartheta+\mathbf{e}_{3} \operatorname{sh} \vartheta \vec{m}\right) \\
& =\mathbf{e}_{1} \operatorname{ctch} 2 \vartheta+\mathbf{e}_{1}(\vec{m} \cdot \vec{r}) \operatorname{sh} 2 \vartheta+\mathbf{e}_{2} \vec{r}-\mathbf{e}_{2} c t \vec{m} \operatorname{sh} 2 \vartheta+\mathbf{e}_{2}(\vec{m} \cdot \vec{r}) \vec{m}(1-\operatorname{ch} 2 \vartheta) .
\end{aligned}
$$

Separating the values with $\mathbf{e}_{1}$ and $\mathbf{e}_{2}$ we get the well known formulas for time and coordinates transformation [25]:

$$
t^{\prime}=\frac{t-x v / c^{2}}{\sqrt{1-v^{2} / c^{2}}}, \quad x^{\prime}=\frac{x-t v}{\sqrt{1-v^{2} / c^{2}}}, y^{\prime}=y, \quad z^{\prime}=z,
$$

where $x$ is the coordinate along the $\vec{m}$ vector.

Let us also consider the Lorentz transformation of the full sedenion $\tilde{\boldsymbol{V}}$. The transformed sedenion $\tilde{\boldsymbol{V}}^{\prime}$ can be written as sedenionic product

$$
\begin{gathered}
\tilde{\boldsymbol{V}}^{\prime}=\tilde{\boldsymbol{L}}^{*} \tilde{\boldsymbol{V}} \tilde{\boldsymbol{L}} . \\
\tilde{\boldsymbol{V}}^{\prime}=\left(\operatorname{ch} \vartheta-\mathbf{e}_{\mathrm{tr}} \operatorname{sh} \vartheta \vec{m}\right)\left(\boldsymbol{V}_{0}+\overrightarrow{\boldsymbol{V}}\right)\left(\operatorname{ch} \vartheta+\mathbf{e}_{\mathbf{t r}} \operatorname{sh} \vartheta \vec{m}\right) \\
=\boldsymbol{V}_{0} \operatorname{ch}^{2} \vartheta+\mathbf{e}_{\mathbf{t r}} \boldsymbol{V}_{0} \mathbf{e}_{\mathbf{r t}} \operatorname{sh}^{2} \vartheta-\left(\mathbf{e}_{\mathbf{t r}} \boldsymbol{V}_{0}-\boldsymbol{V}_{0} \mathbf{e}_{\mathrm{tr}}\right) \vec{m} \operatorname{ch} \vartheta \operatorname{sh} \vartheta+\overrightarrow{\boldsymbol{V}} \operatorname{ch}^{2} \vartheta \\
-\mathbf{e}_{\mathrm{tr}} \overrightarrow{\boldsymbol{m}} \overrightarrow{\boldsymbol{V}} \vec{m} \mathbf{e}_{\mathrm{tr}} \operatorname{sh}^{2} \vartheta-\left(\mathbf{e}_{\mathbf{t r}} \vec{m} \overrightarrow{\boldsymbol{V}}-\overrightarrow{\boldsymbol{V}} \vec{m} \mathbf{e}_{\mathrm{tr}}\right) \operatorname{ch} \vartheta \operatorname{sh} \vartheta .
\end{gathered}
$$

Rewriting the expression (35) with scalar (16) and vector (17) products we get

$$
\begin{aligned}
\tilde{V}^{\prime}= & \boldsymbol{V}_{0} \operatorname{ch}^{2} \vartheta+\mathbf{e}_{\mathbf{t r}} \boldsymbol{V}_{0} \mathbf{e}_{\mathbf{t r}} \operatorname{sh}^{2} \vartheta-\left(\mathbf{e}_{\mathbf{t r}} \boldsymbol{V}_{0}-\boldsymbol{V}_{0} \mathbf{e}_{\mathbf{t r}}\right) \vec{m} \operatorname{ch} \vartheta \operatorname{sh} \vartheta+\overrightarrow{\boldsymbol{V}} \operatorname{ch}^{2} \vartheta-\mathbf{e}_{\mathbf{t r}} \overrightarrow{\boldsymbol{V}} \mathbf{e}_{\mathbf{t r}} \operatorname{sh}^{2} \vartheta-2 \mathbf{e}_{\mathbf{t r}}(\vec{m} \cdot \overrightarrow{\boldsymbol{V}}) \mathbf{e}_{\mathbf{t r}} \vec{m} \operatorname{sh}^{2} \vartheta \\
& -\left(\mathbf{e}_{\mathbf{t r}}(\vec{m} \cdot \overrightarrow{\boldsymbol{V}})-(\overrightarrow{\boldsymbol{V}} \cdot \vec{m}) \mathbf{e}_{\mathbf{t r}}\right) \operatorname{ch} \vartheta \operatorname{sh} \vartheta-\left(\mathbf{e}_{\mathbf{t r}}[\vec{m} \times \overrightarrow{\boldsymbol{V}}]-[\overrightarrow{\boldsymbol{V}} \times \vec{m}] \mathbf{e}_{\mathrm{tr}}\right) \operatorname{ch} \vartheta \operatorname{sh} \vartheta .
\end{aligned}
$$

Thus, the transformed sedenion has the following components: 


$$
\begin{aligned}
& V^{\prime}=V, \\
& V_{\mathbf{t r}}^{\prime}=V_{\mathbf{t r}}, \\
& V_{\mathbf{r}}^{\prime}=V_{\mathbf{r}} \operatorname{ch} 2 \vartheta-\mathbf{e}_{\mathbf{t r}}\left(\vec{m} \cdot \vec{V}_{\mathbf{t}}\right) \operatorname{sh} 2 \vartheta, \\
& V_{\mathbf{t}}^{\prime}=V_{\mathbf{t}} \operatorname{ch} 2 \vartheta-\mathbf{e}_{\mathbf{t r}}\left(\vec{m} \cdot \vec{V}_{\mathbf{r}}\right) \operatorname{sh} 2 \vartheta, \\
& \vec{V}^{\prime}=\vec{V} \operatorname{ch} 2 \vartheta-(\vec{m} \cdot \vec{V}) \vec{m}(1-\operatorname{ch} 2 \vartheta)-\mathbf{e}_{\mathbf{t r}}\left[\vec{m} \times \vec{V}_{\mathrm{rt}}\right] \operatorname{sh} 2 \vartheta, \\
& \vec{V}_{\mathbf{t r}}^{\prime}=\vec{V}_{\mathbf{t r}} \operatorname{ch} 2 \vartheta-\left(\vec{m} \cdot \vec{V}_{\mathbf{t r}}\right) \vec{m}(1-\operatorname{ch} 2 \vartheta)-\mathbf{e}_{\mathrm{tr}}[\vec{m} \times \vec{V}] \operatorname{sh} 2 \vartheta, \\
& \vec{V}_{\mathbf{r}}^{\prime}=\vec{V}_{\mathbf{r}}+\left(\vec{m} \cdot \overrightarrow{V_{\mathbf{r}}}\right) \vec{m}(1-\operatorname{ch} 2 \vartheta)-\mathbf{e}_{\mathbf{t r}} V_{\mathbf{t}} \vec{m} \operatorname{sh} 2 \vartheta, \\
& \vec{V}_{\mathbf{t}}^{\prime}=\vec{V}_{\mathbf{t}}+\left(\vec{m} \cdot \vec{V}_{\mathbf{t}}\right) \vec{m}(1-\operatorname{ch} 2 \vartheta)-\mathbf{e}_{\mathbf{t r}} V_{\mathbf{r}} \vec{m} \operatorname{sh} 2 \vartheta .
\end{aligned}
$$

\section{Subalgebras of Space-Time Complex Numbers, Quaternions and Octonions}

The sedenionic basis introduced above enables constructing different types of low-dimensional hypercomplex numbers. For example, one can introduce space-time complex numbers

$$
\begin{aligned}
& Z_{\mathbf{t}}=Z_{1}+\mathbf{e}_{\mathbf{t}} z_{2}, \\
& Z_{\mathbf{r}}=Z_{1}+\mathbf{e}_{\mathbf{r}} Z_{2}, \\
& Z_{\mathbf{t r}}=Z_{1}+\mathbf{e}_{\mathbf{t r}} Z_{2},
\end{aligned}
$$

where $z_{1}$ and $z_{2}$ real numbers. These values are transformed under space and time conjugation and Lorentz transformations. Moreover, we can consider the space-time quaternions, which differ in their properties with respect to the operations of the spatial and time inversion and Lorentz transformations:

$$
\begin{aligned}
& \widehat{q}=q_{0} \mathbf{a}_{\mathbf{0}}+\mathbf{e}_{\mathbf{0}}\left(q_{1} \mathbf{a}_{\mathbf{1}}+q_{2} \mathbf{a}_{2}+q_{3} \mathbf{a}_{3}\right), \\
& \widehat{q}_{\mathbf{t}}=q_{0} \mathbf{a}_{\mathbf{0}}+\mathbf{e}_{\mathbf{t}}\left(q_{1} \mathbf{a}_{\mathbf{1}}+q_{2} \mathbf{a}_{\mathbf{2}}+q_{3} \mathbf{a}_{3}\right), \\
& \widehat{q}_{\mathbf{r}}=q_{0} \mathbf{a}_{\mathbf{0}}+\mathbf{e}_{\mathbf{r}}\left(q_{1} \mathbf{a}_{\mathbf{1}}+q_{2} \mathbf{a}_{2}+q_{3} \mathbf{a}_{3}\right), \\
& \widehat{q}_{\mathbf{t r}}=q_{0} \mathbf{a}_{\mathbf{0}}+\mathbf{e}_{\mathbf{t r}}\left(q_{1} \mathbf{a}_{\mathbf{1}}+q_{2} \mathbf{a}_{2}+q_{3} \mathbf{a}_{\mathbf{3}}\right) .
\end{aligned}
$$

The absolute quaternion (39) is the sum of the absolute scalar and absolute vector. It remains constant under the transformations of space and time inversion (27). Time quaternion $\hat{q}_{t}$, space quaternion $\hat{q}_{r}$ and space-time quaternion $\hat{q}_{\mathrm{tr}}$ are transformed under inversions in accordance with the commutation rules for the basis elements $\mathbf{e}_{\mathbf{t}}, \mathbf{e}_{\mathbf{r}}, \mathbf{e}_{\mathrm{tr}}$. For example, performing the operation of time inversion (see (27)) with the quaternion $\hat{q}_{\mathbf{t}}$ we obtain the conjugated quaternion

$$
\hat{R}_{\mathbf{t}} \hat{q}_{\mathbf{t}}=-\mathbf{e}_{\mathbf{r}} \widehat{q}_{\mathbf{t}} \mathbf{e}_{\mathbf{r}}=q_{0} \mathbf{a}_{0}-\mathbf{e}_{\mathbf{t}}\left(q_{1} \mathbf{a}_{1}+q_{2} \mathbf{a}_{2}+q_{3} \mathbf{a}_{3}\right) .
$$

In addition, the sedenionic basis allows one to construct various types of space-time eight-component octonions:

$$
\begin{aligned}
& \breve{G}_{\mathbf{t}}=G_{00}+G_{01} \mathbf{a}_{\mathbf{1}}+G_{02} \mathbf{a}_{\mathbf{2}}+G_{03} \mathbf{a}_{3}+\mathbf{e}_{\mathbf{t}} G_{10}+\mathbf{e}_{\mathbf{t}}\left(G_{11} \mathbf{a}_{\mathbf{1}}+G_{12} \mathbf{a}_{2}+G_{13} \mathbf{a}_{3}\right), \\
& \breve{G}_{\mathbf{r}}=G_{00}+G_{01} \mathbf{a}_{\mathbf{1}}+G_{02} \mathbf{a}_{2}+G_{03} \mathbf{a}_{\mathbf{3}}+\mathbf{e}_{\mathbf{r}} G_{20}+\mathbf{e}_{\mathbf{r}}\left(G_{21} \mathbf{a}_{\mathbf{1}}+G_{22} \mathbf{a}_{\mathbf{2}}+G_{23} \mathbf{a}_{3}\right), \\
& \breve{G}_{\mathbf{t r}}=G_{00}+G_{01} \mathbf{a}_{\mathbf{1}}+G_{02} \mathbf{a}_{2}+G_{03} \mathbf{a}_{3}+\mathbf{e}_{\mathbf{t r}} G_{30}+\mathbf{e}_{\mathbf{t r}}\left(G_{31} \mathbf{a}_{\mathbf{1}}+G_{32} \mathbf{a}_{2}+G_{33} \mathbf{a}_{3}\right) .
\end{aligned}
$$

\section{Generalized Sedenionic Equations of Relativistic Quantum Mechanics}

The wave function of free quantum particle should satisfy an equation, which is obtained from the Einstein relation for energy and momentum

$$
E^{2}-c^{2} p^{2}=m_{0}^{2} c^{4},
$$


by means of changing classical energy $E$ and momentum $\vec{p}$ on corresponding quantum-mechanical operators:

$$
\hat{E}=i \hbar \frac{\partial}{\partial t} \text { and } \overrightarrow{\hat{p}}=-i \hbar \vec{\nabla} \text {. }
$$

Here $c$ is the speed of light, $m_{0}$ is the particle rest mass, $\hbar$ is the Planck constant. In sedenion algebra the Einstein relation (47) can be written as

$$
\left(\mathbf{e}_{\mathbf{t}} E+\mathbf{e}_{\mathbf{r}} c \vec{p}+i \mathbf{e}_{\mathrm{tr}} m_{0} c^{2}\right)\left(\mathbf{e}_{\mathbf{t}} E+\mathbf{e}_{\mathbf{r}} c \vec{p}+i \mathbf{e}_{\mathrm{tr}} m_{0} c^{2}\right)=0 .
$$

Let us consider the wave function in the form of space-time sedenion

$$
\tilde{W}(t, \vec{r})=W_{0}(t, \vec{r})+\vec{W}(t, \vec{r}) .
$$

Then the generalized sedenionic wave equation for free particle can be written in the following symmetric form:

$$
\left(\mathbf{e}_{\mathbf{t}} \frac{1}{c} \frac{\partial}{\partial t}-\mathbf{e}_{\mathbf{r}} \vec{\nabla}+\mathbf{e}_{\mathrm{tr}} \frac{m_{0} c}{\hbar}\right)\left(\mathbf{e}_{\mathbf{t}} \frac{1}{c} \frac{\partial}{\partial t}-\mathbf{e}_{\mathbf{r}} \vec{\nabla}+\mathbf{e}_{\mathrm{tr}} \frac{m_{0} c}{\hbar}\right) \tilde{W}=0 .
$$

Note that for electrically charged particle in an external electromagnetic field we have the following sedenionic wave equation:

$$
\left(\mathbf{e}_{\mathbf{t}} \frac{1}{c} \frac{\partial}{\partial t}+\mathbf{e}_{\mathbf{t}} \frac{i e}{\hbar c} \varphi-\mathbf{e}_{\mathbf{r}} \vec{\nabla}+\mathbf{e}_{\mathbf{r}} \frac{i e}{\hbar c} \vec{A}+\mathbf{e}_{\mathbf{t r}} \frac{m_{0} c}{\hbar}\right)\left(\mathbf{e}_{\mathbf{t}} \frac{1}{c} \frac{\partial}{\partial t}+\mathbf{e}_{\mathbf{t}} \frac{i e}{\hbar c} \varphi-\mathbf{e}_{\mathbf{r}} \vec{\nabla}+\mathbf{e}_{\mathbf{r}} \frac{i e}{\hbar c} \vec{A}+\mathbf{e}_{\mathbf{t r}} \frac{m_{0} c}{\hbar}\right) \tilde{W}=0 .
$$

This equation describes the particles with spin $1 / 2$ in an external electromagnetic field [18] [21].

There is a special class of particles described by the first-order wave equation [26]. For these particles the sedenionic Dirac-like wave equation has the following form:

$$
\left(\mathbf{e}_{\mathbf{t}} \frac{1}{c} \frac{\partial}{\partial t}-\mathbf{e}_{\mathbf{r}} \vec{\nabla}+\mathbf{e}_{\mathbf{t r}} \frac{m_{0} c}{\hbar}\right) \tilde{W}=0 .
$$

In fact, this equation describes the special quantum field with zero field strengths [19]. Analogously the electrically charged particle interacting with external electromagnetic field is described by the following sedenionic first-order wave equation:

$$
\left(\mathbf{e}_{\mathbf{t}} \frac{1}{c} \frac{\partial}{\partial t}+\mathbf{e}_{\mathbf{t}} \frac{i e}{\hbar c} \varphi-\mathbf{e}_{\mathbf{r}} \vec{\nabla}+\mathbf{e}_{\mathbf{r}} \frac{i e}{\hbar c} \vec{A}+\mathbf{e}_{\mathbf{t r}} \frac{m_{0} c}{\hbar}\right) \tilde{W}=0 .
$$

This equation also describes particles with spin $1 / 2$ in an external electromagnetic field [19].

\section{Generalized Sedenionic Equations for Massive Field}

The generalized sedenionic wave equation

$$
\left(\mathbf{e}_{\mathbf{t}} \frac{1}{c} \frac{\partial}{\partial t}-\mathbf{e}_{\mathbf{r}} \vec{\nabla}+\mathbf{e}_{\mathbf{t r}} \frac{m_{0} c}{\hbar}\right)\left(\mathbf{e}_{\mathbf{t}} \frac{1}{c} \frac{\partial}{\partial t}-\mathbf{e}_{\mathbf{r}} \vec{\nabla}+\mathbf{e}_{\mathbf{t r}} \frac{m_{0} c}{\hbar}\right) \tilde{W}=0,
$$

enables another interpretation. It can be considered as the equation for the force massive field [27]. In this case the parameter $m_{0}$ is the mass of quantum of field and $\tilde{W}$ is field potential. Considering the phenomenological source of field $\tilde{\boldsymbol{J}}$ we can propose the following nonhomogeneous wave equation for the field potential:

$$
\left(\mathbf{e}_{\mathbf{t}} \frac{1}{c} \frac{\partial}{\partial t}-\mathbf{e}_{\mathbf{r}} \vec{\nabla}+\mathbf{e}_{\mathbf{t r}} \frac{m_{0} c}{\hbar}\right)\left(\mathbf{e}_{\mathbf{t}} \frac{1}{c} \frac{\partial}{\partial t}-\mathbf{e}_{\mathbf{r}} \vec{\nabla}+\mathbf{e}_{\mathbf{t r}} \frac{m_{0} c}{\hbar}\right) \tilde{\boldsymbol{W}}=\tilde{\boldsymbol{J}} .
$$

Seemingly this equation describes the baryon (strong) field [23] [24] and $\tilde{\boldsymbol{J}}$ is baryon current. On the other hand, corresponding nonhomogeneous first-order equation

$$
\left(\mathbf{e}_{\mathbf{t}} \frac{1}{c} \frac{\partial}{\partial t}-\mathbf{e}_{\mathbf{r}} \vec{\nabla}+\mathbf{e}_{\mathbf{t r}} \frac{m_{0} c}{\hbar}\right) \tilde{\boldsymbol{W}}=\tilde{\boldsymbol{I}},
$$

describes the lepton (weak) field, where $\tilde{\boldsymbol{I}}$ is a lepton current [23] [24]. 


\section{Generalized Sedenionic Equations for Massless Field}

In the special case, when the mass of quantum $m_{0}$ is equal to zero, the Equation (56) coincides with the equation for electromagnetic field. Indeed, choosing the potential as

$$
\tilde{W}=\mathbf{e}_{\mathbf{t}} \varphi+\mathbf{e}_{\mathbf{r}} \vec{A}
$$

and the source of field as

$$
\tilde{\boldsymbol{J}}=-\mathbf{e}_{\mathbf{t}} 4 \pi \rho-\mathbf{e}_{\mathbf{r}} \frac{4 \pi}{C} \vec{j},
$$

we obtain the following wave equation:

$$
\left(\mathbf{e}_{\mathbf{t}} \frac{1}{c} \frac{\partial}{\partial t}-\mathbf{e}_{\mathbf{r}} \vec{\nabla}\right)\left(\mathbf{e}_{\mathbf{t}} \frac{1}{c} \frac{\partial}{\partial t}-\mathbf{e}_{\mathbf{r}} \vec{\nabla}\right)\left(\mathbf{e}_{\mathbf{t}} \varphi+\mathbf{e}_{\mathbf{r}} \vec{A}\right)=-\mathbf{e}_{\mathbf{t}} 4 \pi \rho-\mathbf{e}_{\mathbf{r}} \frac{4 \pi}{c} \vec{j} .
$$

After the action of the first operator in the left-hand side of Equation (60) we obtain

$$
\left(\mathbf{e}_{\mathbf{t}} \frac{1}{c} \frac{\partial}{\partial t}-\mathbf{e}_{\mathbf{r}} \vec{\nabla}\right)\left(\mathbf{e}_{\mathbf{t}} \varphi+\mathbf{e}_{\mathbf{r}} \vec{A}\right)=-\frac{1}{c} \frac{\partial \varphi}{\partial t}+\mathbf{e}_{\mathbf{t r}} \frac{1}{c} \frac{\partial \vec{A}}{\partial t}+\mathbf{e}_{\mathbf{t r}} \vec{\nabla} \varphi+(\vec{\nabla} \cdot \vec{A})+[\vec{\nabla} \times \vec{A}] .
$$

In sedenionic algebra the electric and magnetic fields are defined as

$$
\begin{aligned}
& \vec{E}=-\frac{1}{c} \frac{\partial \vec{A}}{\partial t}-\vec{\nabla} \varphi, \\
& \vec{H}=[\vec{\nabla} \times \vec{A}] .
\end{aligned}
$$

Besides we can define the scalar field

$$
f=-\frac{1}{c} \frac{\partial \varphi}{\partial t}+(\vec{\nabla} \cdot \vec{A})
$$

Assuming electric charge conservation the scalar field $f$ can be chosen equal to zero, that coincides with Lorentz gauge condition [22]. In Lorentz gauge we can rewrite the expression (61) as

$$
\left(\mathbf{e}_{\mathbf{t}} \frac{1}{c} \frac{\partial}{\partial t}-\mathbf{e}_{\mathbf{r}} \vec{\nabla}\right)\left(\mathbf{e}_{\mathbf{t}} \varphi+\mathbf{e}_{\mathbf{r}} \vec{A}\right)=-\mathbf{e}_{\mathbf{t r}} \vec{E}+\vec{H} .
$$

Then the wave Equation (60) can be represented in the following form:

$$
\left(\mathbf{e}_{\mathbf{t}} \frac{1}{c} \frac{\partial}{\partial t}-\mathbf{e}_{\mathbf{r}} \vec{\nabla}\right)\left(-\mathbf{e}_{\mathbf{t r}} \vec{E}+\vec{H}\right)=-\mathbf{e}_{\mathbf{t}} 4 \pi \rho-\mathbf{e}_{\mathbf{r}} \frac{4 \pi}{c} \vec{j}
$$

Performing sedenionic multiplication in the left-hand side of Equation (65) we get

$$
\mathbf{e}_{\mathbf{r}} \frac{1}{c} \frac{\partial \vec{E}}{\partial t}+\mathbf{e}_{\mathbf{t}}(\vec{\nabla} \cdot \vec{E})+\mathbf{e}_{\mathbf{t}}[\vec{\nabla} \times \vec{E}]+\mathbf{e}_{\mathbf{t}} \frac{1}{c} \frac{\partial \vec{H}}{\partial t}-\mathbf{e}_{\mathbf{r}}(\vec{\nabla} \cdot \vec{H})-\mathbf{e}_{\mathbf{r}}[\vec{\nabla} \times \vec{H}]=-\mathbf{e}_{\mathbf{t}} 4 \pi \rho-\mathbf{e}_{\mathbf{r}} \frac{4 \pi}{c} \vec{j}
$$

Separating space-time values we obtain the system of equations in the following form:

$$
\begin{array}{ll}
\mathbf{e}_{\mathbf{t}}(\vec{\nabla} \cdot \vec{E})=-\mathbf{e}_{\mathbf{t}} 4 \pi \rho, & \text { (time scalar part) } \\
\mathbf{e}_{\mathbf{r}}[\vec{\nabla} \times \vec{E}]=\mathbf{e}_{\mathbf{r}} \frac{1}{c} \frac{\partial \vec{E}}{\partial t}+\mathbf{e}_{\mathbf{r}} \frac{4 \pi}{c} \vec{j}, & \text { (space vector part) } \\
\mathbf{e}_{\mathbf{t}}[\vec{\nabla} \times \vec{E}]=-\mathbf{e}_{\mathbf{t}} \frac{1}{c} \frac{\partial \vec{H}}{\partial t}, & \text { (time vector part) } \\
\mathbf{e}_{\mathbf{r}}(\vec{\nabla} \cdot \vec{H})=0 . & \text { (space scalar part) }
\end{array}
$$

The system (67) coincides with the Maxwell equations.

Among the solutions of the homogeneous sedeonic wave equation of electromagnetic field (60) there is a special class that satisfies the sedeonic first-order equation of the following form [22]:

$$
\left(\mathbf{e}_{\mathbf{t}} \frac{1}{c} \frac{\partial}{\partial t}-\mathbf{e}_{\mathbf{r}} \vec{\nabla}\right) \tilde{W}_{v}=0 \text {. }
$$


This equation describes the free neutrino field. On the other hand, let us consider the nonhomogeneous equation of neutrino field

$$
\left(\mathbf{e}_{\mathrm{t}} \frac{1}{c} \frac{\partial}{\partial t}-\mathbf{e}_{\mathrm{r}} \vec{\nabla}\right) \tilde{\boldsymbol{W}}_{v}=\tilde{\boldsymbol{I}}_{v},
$$

where $\tilde{\boldsymbol{I}}_{v}$ is phenomenological source. We choose the scalar source in the form

$$
\tilde{\boldsymbol{I}}_{v}=4 \pi \sigma_{v},
$$

where $\sigma_{v}$ is the density of neutrino charge. Choosing the potential $\tilde{W}_{v}$ in the form (58):

$$
\tilde{W}_{v}=i \mathbf{e}_{\mathrm{t}} \varphi_{v}+\mathbf{e}_{\mathrm{r}} \vec{A}_{v},
$$

we obtain following nonhomogeneous equation for the neutrino field:

$$
\left(\mathbf{e}_{\mathrm{t}} \frac{1}{c} \frac{\partial}{\partial t}-\mathbf{e}_{\mathrm{r}} \vec{\nabla}\right)\left(\mathbf{e}_{\mathrm{t}} \varphi_{v}+\mathbf{e}_{\mathrm{r}} \overrightarrow{A_{v}}\right)=4 \pi \sigma_{v} .
$$

It follows that in this case only scalar field strength $f_{v}$ (see (63)) is nonzero:

$$
f_{v}=4 \pi \sigma_{v} .
$$

The density of neutrino charge for point source is equal

$$
\sigma_{v}=q_{v} \delta(\vec{r}),
$$

where $q_{v}$ is point neutrino charge. Then the interaction energy of two point neutrino charges can be represented as follows:

$$
W_{v 1 v 2}=\frac{1}{8 \pi} \int f_{v 1} f_{v 2} \mathrm{~d} V
$$

Substituting (73) and (74), we obtain

$$
W_{v 1 v 2}=2 \pi q_{v 1} q_{v 2} \delta(\vec{R}),
$$

where $\vec{R}$ is the vector of distance between first and second charges.

\section{Discussion}

The algebra of sedenions proposed in this article is the anticommutative associative space-time Clifford algebra. The sedenionic basis elements $\mathbf{a}_{\mathbf{n}}$ are responsible for the spatial rotation, while the elements $\mathbf{e}_{\mathbf{n}}$ are responsible for the space-time inversions. Mathematically, these two bases are equivalent, and the different physical properties attributed to them are an important physical essence of our sedenionic hypothesis.

In contrast to the previously discussed sedeonic algebra [20]-[23], which uses the multiplication rules of basic elements $\mathbf{a}_{\mathbf{n}}^{\prime}$ and $\mathbf{e}_{\mathbf{n}}^{\prime}$ proposed by A. Macfarlane [28], the multiplication rules for sedenionic basis elements $\mathbf{a}_{\mathbf{n}}$ and $\mathbf{e}_{\mathbf{n}}$ coincide with the rules for quaternion units introduced by W. R. Hamilton [29]. There is a close connection between these two basses. The transition from the sedeonic basis to sedenionic basis is performed by following replacement:

$$
\begin{aligned}
& \mathbf{a}_{\mathbf{n}}^{\prime}=i \mathbf{a}_{\mathbf{n}}, \\
& \mathbf{e}_{\mathbf{n}}^{\prime}=i \mathbf{e}_{\mathbf{n}} .
\end{aligned}
$$

There is one disadvantage of sedenions connected with the fact that the square of the vector is a negative value. However, on the other side the sedenionic rules of cross-multiplying do not contain the imaginary unit and this leads to the some simplifications in the calculations. But of course, the physical results do not depend on the choice of algebra, so these two algebras are equivalent.

\section{Conclusion}

Thus, in this paper we presented the sixteen-component hypercomplex values sedenions, generating associative 
noncommutative space-time algebra. We considered the generalization of the relativistic quantum mechanics and theory of massive and massless fields based on hypercomplex scalar-vector wave functions and sedenionic space-time operators.

\section{Acknowledgements}

The authors are very thankful to G. V. Mironova for kind assistance and moral support.

\section{References}

[1] Adler, S.L. (1995) Quaternionic Quantum Mechanics and Quantum Fields. Oxford University Press, New York.

[2] Imaeda, K. (1976) A New Formulation of Classical Electrodynamics. Nuovocimento, 32, 138-162. http://dx.doi.org/10.1007/BF02726749

[3] Majernik, V. (1999) Quaternionic Formulation of the Classical Fields. Advances in Applied Clifford Algebras, 9, 119130. http://dx.doi.org/10.1007/BF03041944

[4] Davies, A.J. (1990) Quaternionic Dirac Equation. Physical Review D, 41, 2628-2630. http://dx.doi.org/10.1103/PhysRevD.41.2628

[5] Schwartz, C. (2006) Relativistic Quaternionic Wave Equation. Journal of Mathematical Physics, 47, Article ID: 122301. http://dx.doi.org/10.1063/1.2397555

[6] Liu, Y.-F. (2002) Triality, Biquaternion and Vector Representation of the Dirac Equation. Advances in Applied Clifford Algebras, 12, 109-124. http://dx.doi.org/10.1007/BF03161242

[7] Gogberashvili, M. (2006) Octonionic Electrodynamics. Journal of Physics A: Mathematics in General, 39, 7099-7104. http://dx.doi.org/10.1088/0305-4470/39/22/020

[8] Gamba, A. (1998) Maxwell's Equations in Octonion Form. NuovoCimento A, 111, 293-302.

[9] Tolan, T., Özdas, K. and Tanisli, M. (2006) Reformulation of Electromagnetism with Octonions. Nuovo Cimento B, 121, 43-55. http://dx.doi.org/10.1393/ncb/i2005-10189-9

[10] Gogberashvili, M. (2006) Octonionic Version of Dirac Equations. International Journal of Modern Physics A, 21, 3513-3523. http://dx.doi.org/10.1142/S0217751X06028436

[11] De Leo, S. and Abdel-Khalek, K. (1996) Octonionic Dirac Equation. Progress in Theoretical Physics, 96, 833-846. http://dx.doi.org/10.1143/PTP.96.833

[12] Dickson, L.E. (1919) On Quaternions and Their Generalization and the History of the Eight Square Theorem. Annals of Mathematics, 20, 155-171. http://dx.doi.org/10.2307/1967865

[13] Imaeda, K. and Imaeda, M. (2000) Sedenions: Algebra and Analysis. Applied Mathematics and Computations, 115, 7788. http://dx.doi.org/10.1016/S0096-3003(99)00140-X

[14] Carmody, K. (1988) Circular and Hyperbolic Quaternions, Octonions, and Sedenions. Applied Mathematics and Computations, 28, 47-72. http://dx.doi.org/10.1016/0096-3003(88)90133-6

[15] Carmody, K. (1997) Circular and Hyperbolic Quaternions, Octonions, and Sedenions-Further Results. Applied Mathematics and Computations, 84, 27-47. http://dx.doi.org/10.1016/S0096-3003(96)00051-3

[16] Köplinger, J. (2006) Dirac Equation on Hyperbolic Octonions. Applied Mathematics and Computations, 182, 443-446. http://dx.doi.org/10.1016/j.amc.2006.04.005

[17] Mironov, V.L. and Mironov, S.V. (2009) Octonic Representation of Electromagnetic Field Equations. Journal of Mathematical Physics, 50, Article ID: 012901. http://dx.doi.org/10.1063/1.3041499

[18] Mironov, V.L. and Mironov, S.V. (2009) Octonic Second-Order Equations of Relativistic Quantum Mechanics. Journal of Mathematical Physics, 50, Article ID: 012302. http://dx.doi.org/10.1142/S0217751X09045480

[19] Mironov, V.L. and Mironov, S.V. (2009) Octonic First-Order Equations of Relativistic Quantum Mechanics. International Journal of Modern Physics A, 24, 4157-4167. http://dx.doi.org/10.1142/S0217751X09045480

[20] Mironov, V.L. and Mironov, S.V. (2011) Noncommutative Sedeons and Their Application in Field Theory. e-Print. http://arxiv.org/abs/1111.4035

[21] Mironov, V.L. and Mironov, S.V. (2013) Reformulation of Relativistic Quantum Mechanics Equations with NonCommutative Sedeons. Applied Mathematics, 4, 53-60. http://dx.doi.org/10.4236/am.2013.410A3007

[22] Mironov, V.L. and Mironov, S.V. (2014) Sedeonic Equations of Gravitoelectromagnetism. Journal of Modern Physics, 5, 917-927. http://dx.doi.org/10.4236/jmp.2014.510095

[23] Mironov, S.V. and Mironov, V.L. (2014) Sedeonic Equations of Massive Fields. International Journal of Theoretical 
Physics, e-Print. http://vixra.org/abs/1311.0005

[24] Mironov, S.V. and Mironov, V.L. (2014) Space-Time Sedeons and Their Application in Relativistic Quantum Mechanics and Field Theory. Institute for Physics of Microstructures RAS, Nizhny Novgorod. http://vixra.org/abs/1407.0068

[25] Landau, L.D. and Lifshits, E.M. (1975) Classical Theory of Fields. 4th Edition, Pergamon Press, New York.

[26] Dirac, P.A.M. (1958) The Principles of Quantum Mechanics. Clarendon Press, Oxford.

[27] Cafaro, C. and Ali, S.A. (2006) The Spacetime Algebra Approach to Massive Classical Electrodynamics with Magnetic Monopoles. Advances in Applied Clifford Algebras, 17, 23-36. http://dx.doi.org/10.1007/s00006-006-0014-7

[28] Macfarlane, A. (1900) Hyperbolic Quaternions. Proceedings of the Royal Society at Edinburgh, 1899-1900 Session, 169-181.

[29] Hamilton, W.R. (1853) Lectures on Quaternions. Royal Irish Academy, Dublin. 
Scientific Research Publishing (SCIRP) is one of the largest Open Access journal publishers. It is currently publishing more than 200 open access, online, peer-reviewed journals covering a wide range of academic disciplines. SCIRP serves the worldwide academic communities and contributes to the progress and application of science with its publication.

Other selected journals from SCIRP are listed as below. Submit your manuscript to us via either submit@scirp.org or Online Submission Portal.
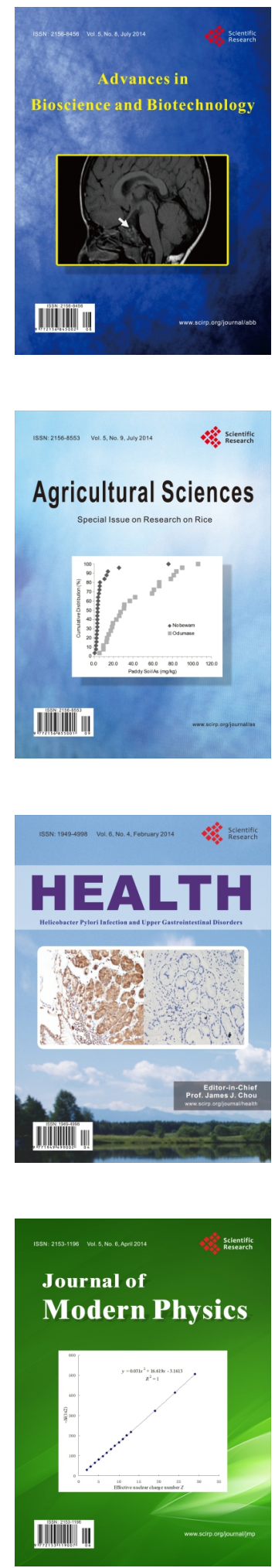
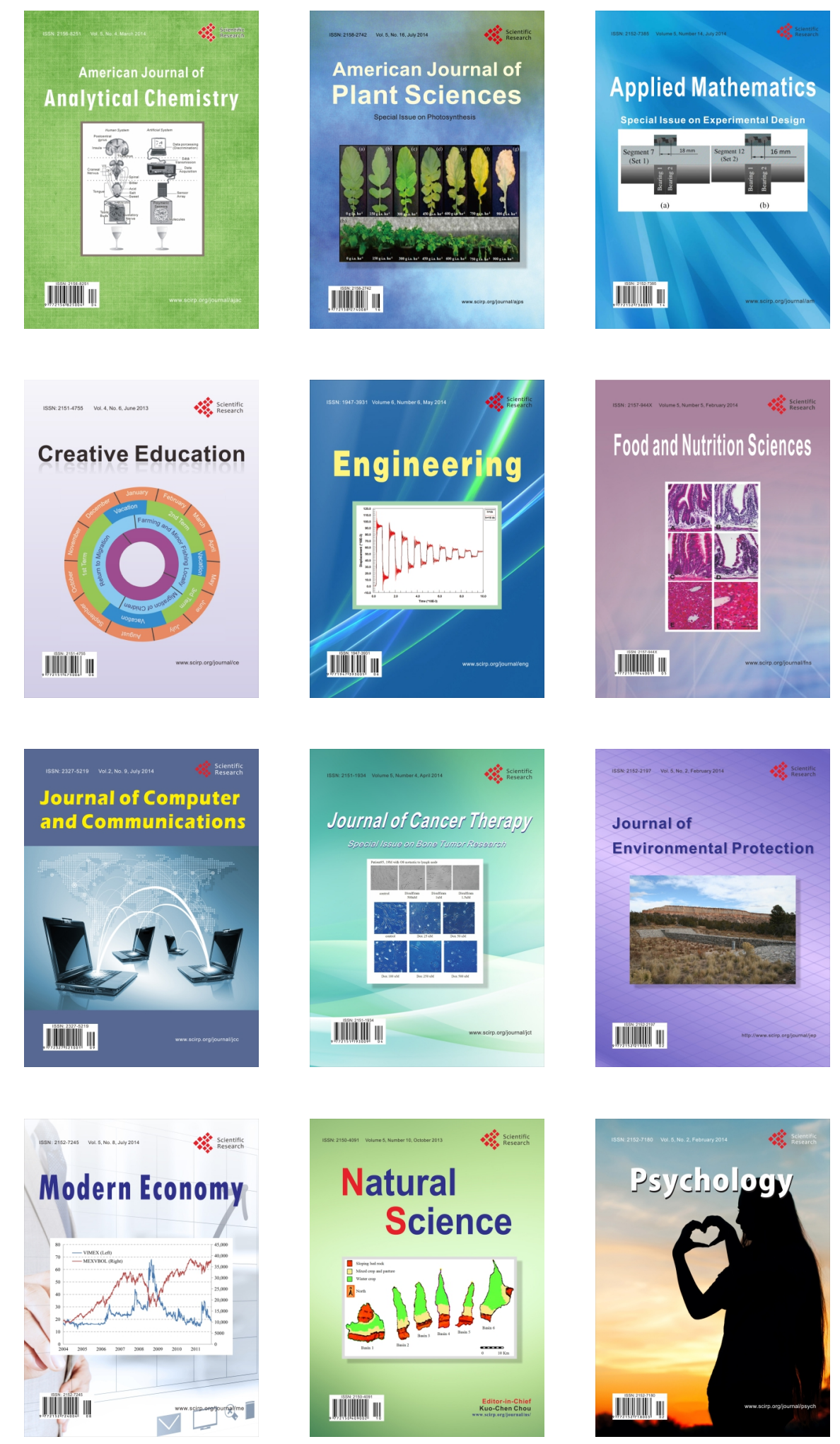